\title{
ACIDENTES COM MATERIAL BIOLÓGICO EM SERVIÇOS DE URGÊNCIA E EMERGÊNCIA
}

\author{
ACCIDENTS WITH BIOLOGICAL MATERIAL IN URGENCY \\ AND EMERGENCY SERVICES
}

\section{ACCIDENTES CON MATERIAL BIOLÓGICO EN LOS SERVICIOS DE URGENCIA Y EMERGENCIA}

\author{
Katiane Martins Mendonça* \\ Anaclara Ferreira Veiga Tipple ${ }^{* *}$ \\ Adenícia Custódia Silva e Sousa ${ }^{* * *}$ \\ Milca Severino Pereira ${ }^{* * *}$ \\ CRISTIANE RAPPARINI ${ }^{* * * * *}$
}

\begin{abstract}
RESUMO
Objetivo: Analisar casos de acidentes com material biológico entre profissionais da equipe de enfermagem, em setores de urgência e emergência. Material e método: Pesquisa exploratória, que ocorreu entre maio a julho de 2009, com aprovação por Comitês de Ética (No 065/08, $12 / 08$ e 118/08). A coleta de dados do referido estudo foi realizada por meio de observação direta, sem, com preenchimento de dois check-list. Resultados: Aconteceram oito acidentes, sendo que a maioria foi por exposição de sangue nas mãos dos trabalhadores e três envolveram vômito. O não uso dos equipamentos de proteção em todos os casos mostra uma subvalorização dessas barreiras por parte dos trabalhadores e ainda dos gerentes, já que nem todos estavam disponíveis. Nenhum acidente foi notificado. Conclusão: $O$ processo de trabalho nos setores de urgência e emergência requer um olhar diferenciado, que considere as suas especificidades, para o planejamento e implantação de uma cultura de segurança frente ao risco biológico.
\end{abstract}

Palavras chave: Precauções universais, equipe de enfermagem, exposição ocupacional, exposição a agentes biológicos, enfermagem em emergência.

\begin{abstract}
Objective: To analyse cases of biological material accident among nursing staff in emergency areas. Method: Exploratory research held May and July 2009, with approval by the Ethics Committee (No 065/08, 12/08 and 118/08). Two checklist were used for data collection. Results: Eight accidents occurred, and the most involved

\footnotetext{
* Enfermeira. Doutora em Enfermagem. Docente do Instituto Federal de Educação, Ciência e Tecnologia de Goiás. Campus Goiânia Oeste. Goiânia-Goiás-Brasil. Email: katiane2303@gmail.com

${ }^{* *}$ Enfermeira. Doutora em Enfermagem. Docente da Faculdade de Enfermagem da Universidade Federal de Goiás. Goiânia-Goiás-Brasil. Email: anaclara.fen@gmail.com

${ }^{* * *}$ Enfermeira. Doutora em Enfermagem. Coordenadora do Programa de Mestrado em Atenção à Saúde da Pontifícia Universidade Católica de Goiás. Goiânia-Goiás-Brasil. Email: adeniciafen@gmail.com

${ }_{* * * *}^{*}$ Enfermeira. Doutora em Enfermagem. Pró-Reitora de Pós-Graduação e Pesquisa da Pontifícia Universidade Católica de Goiás. Goiânia-Goiás-Brasil. Email: milcaseverino@gmail.com

${ }_{* * * * *}$ Médica. Doutora em infectologia. Coordenadora do Projeto Riscobiologico.org. Email: rapparini@riscobiologico.org
} 
blood in the hands of healthcare workers and three involved vomiting. The non-use of protective equipment in all the cases shows an underestimation of these barriers by healthcare workers and by managers, since not all were available. No accident was reported. Conclusion: The work process in the emergency areas requires a different look, considering their specificities for planning and implementation of a safe culture against the biological risk.

Key words: Universal precautions, nursing, team, occupational exposure, exposure to biological agents, emergency nursing

\section{RESUMEN}

Objetivo: Analizar casos de accidente a material biológico entre trabajadores de enfermería en las áreas de urgencia y de emergencia. Método: Investigación exploratoria, ocurrió en mayo y julio de 2009, con la aprobación del Comité de Ética (No 065/08, 12/08 y 118/08). La recolección de los datos fue realizada por medio de la observación directa, utilizando dos check-list. Resultados: Se produjeron ocho accidentes, y la mayoría fue con sangre en las manos de los trabajadores y tres tenían vómito. La no utilización de equipos de protección en todos los accidentes se muestra como una subestimación de estas barreras por los trabajadores y por los directores, ya que pocos estaban disponibles. Ningún accidente fue reportado. El proceso de trabajo en las áreas de emergencia requiere una mirada diferente, que considere sus características específicas para planos e implementación de una cultura de seguridad contra el riesgo biológico.

Palabras clave: Precauciones universales, grupo de enfermería, exposición profesional, exposición a agentes biológicos, enfermería de urgencia.

Fecha recepción: 06/06/13. Fecha acepctación: 03/04/14.

\section{INTRODUÇÃO}

Sabe-se que as especificidades de cada setor em uma instituição de saúde podem potencializar os riscos ocupacionais. Um exemplo é o setor de urgência e emergência, caracterizado pela marcante complexidade e peculiaridade do cuidado e pela alta ocorrência de exposições ocupacionais envolvendo Material Biológico (MB) (1-3).

Essas exposições recebem destaque na literatura mundial, especialmente no que se refere à assistência prestada pela equipe de enfermagem. Mas, concomitante à elevada taxa de ocorrência, estima-se a coexistência de alta taxa de subnotificação (4-5). Fator que dificulta ou impossibilita o planejamento e a implementação de estratégias de intervenção preventivas.

Diversas abordagens metodológicas são utilizadas, mundialmente, para investigar a ocorrência de exposições a MB, como a caracterização dos sujeitos; a prevalência e perfil das exposições; recursos humanos, materiais e estrutura-física envolvida, além de dados comportamentais (6). Nas investigações sobre exposições ocupacionais a MB, entre a equipe de enfermagem, predominam estudos com abordagem quantitativa, do tipo corte transversal, com dados coletados por meio de questionários e análise de prontuários (7).

O método observacional mostra-se relevante, principalmente, para entender o que os indivíduos fazem e sob quais circunstâncias. Os dados coletados a partir da observação fornecem subsídios para diagnosticar uma situação problema, facilitar a escolha das técnicas e procedimentos empregados na pesquisa e na avaliação da sua eficácia (8).

A iniciativa Strengthening the Reporting of Observational studies in Epidemiology ("Stro- 
be - Aprimorando a Apresentação de Resultados de Estudos Observacionais em Epidemiologia") aparece no cenário mundial para melhorar a qualidade da descrição de estudos observacionais e assim, facilitar a leitura crítica dessas publicações (9-10).

Nesse sentido este artigo poderá contribuir para o conhecimento das exposições envolvendo $\mathrm{MB}$ em setores de urgência e emergência, e assim fornecer subsídios para profissionais de serviços de controle de infecção e de programas de educação permanentes para a minimização do risco biológico nesses locais e para gestores e pesquisadores da área da saúde a fim de vislumbrarem nas investigações observacionais, a oportunidade de apresentar o que realmente acontece no cotidiano laboral de profissionais da saúde -fragilidades e potencialidades- relacionadas à assistência e à gerência na saúde. Além disso, contribuições para o ensino de enfermagem para que a dicotomia entre a teoria e a prática seja minimizada e para que o futuro profissional incorpore no trabalho as medidas preventivas imprescindíveis para uma atuação segura. $\mathrm{O}$ objetivo desse relato é analisar casos de acidentes com MB entre profissionais da equipe de enfermagem, em setores de urgência e emergência.

\section{MATERIAL E MÉTODO}

Trata-se de uma pesquisa exploratória a qual identificou como achados de pesquisa casos de acidentes com MB durante a realização da coleta de dados, que ocorreu entre maio a julho de 2009 para fins de desenvolvimento de uma dissertação de Mestrado (aprovado pelos Comitês de Ética em Pesquisa das três instituições, protocolos No 065/08, 12/08 e $118 / 08$ ) e todos os participantes assinaram o termo de consentimento, apresentado juntamente com os objetivos do estudo, após o período de observação.

A coleta de dados do referido estudo foi realizada por meio de observação direta, sem interferência do pesquisador, com preenchimento de dois check-list, sendo um para avaliação da estrutura física, de recursos materiais e humanos disponíveis para o cumprimento das Precauções Padrão e outro para avaliar comportamentos dos profissionais para prevenir/minimizar o risco biológico, no preparo e na administração de medicamentos, e que se relacionam com o risco ocupacional e do cliente: higienização de mãos, equipamentos de proteção, manejo de resíduos (foco nos grupos A4 e E) e manutenção da cadeia asséptica. Os sujeitos do estudo foram 130 profissionais da equipe de enfermagem que atuavam no preparo e na administração de medicamentos, dos serviços de urgência e emergência, dos três hospitais públicos, de grande porte, da cidade de Goiânia-GO.

A coleta de dados foi realizada, exclusivamente, por uma das pesquisadoras, que não apresentava vínculos com as instituições e observou todos os profissionais de enfermagem envolvidos nas etapas finais do sistema de medicação, de forma individual em todos os turnos. O período de observação não foi pré-estabelecido, mas buscou-se observar todos os profissionais que atuavam nas etapas finais do sistema de medicação nos locais do estudo e dessa forma foram realizadas $24 \mathrm{~h}$ de observação semanais.

\section{RESULTADOS}

Foram observadas oito acidentes com MB, entre 130 profissionais da equipe de enfermagem, na realização de 292 procedimentos de preparo e administração de medicamentos. Houve 31 horas de observação para 36 procedimentos de preparo e administração de medicamentos. Todos os acidentes ocorreram durante punções venosas. A Tabela 1 apresenta a caracterização dos casos.

Nenhum acidente foi comunicado ou no- 
Tabela 1. Caracterização de casos observados de acidentes com Material Biológico $(\mathrm{N}=8)$, nas etapas finais do sistema de medicação de serviços de urgência e emergência de Goiânia-GO. 2009.

\begin{tabular}{|c|c|c|c|}
\hline Caso & Material Biológico & Local do corpo atingido & Conduta pós-exposição \\
\hline 1 & Sangue & Mãos & Lavou com água e sabão \\
\hline 2 & Sangue & Mãos & Lavou com água e sabão \\
\hline 3 & Sangue & Mãos & Lavou com água e sabão \\
\hline 4 & Sangue & Mãos & $\begin{array}{l}\text { Lavou com água e sabão e, em seguida, } \\
\text { realizou antissepsia com álcool a 70\% }\end{array}$ \\
\hline 5 & Sangue & Mãos & $\begin{array}{l}\text { Lavou com água e sabão e, em seguida, } \\
\text { realizou antissepsia com álcool a 70\% }\end{array}$ \\
\hline 6 & Vômito & Membros superiores e pés & Lavou com soro fisiológico \\
\hline 7 & Vômito & Membros superiores e pés & Lavou com água e sabão \\
\hline 8 & $\begin{array}{l}\text { Vômito e secreção } \\
\text { pulmonar* }\end{array}$ & $\begin{array}{l}\text { Membros superiores e face } \\
\text { (mucosa ocular) }\end{array}$ & Enxaguou com água \\
\hline
\end{tabular}

* Durante episódio de tosse produtiva, o paciente apresentou o episódio de vômito.

tificado ao responsável pela unidade e nem ao serviço, oficialmente, encarregado por esse registro. Todos os profissionais continuaram o atendimento. No caso 8, o profissional apresentava pele não íntegra (cortes na região de polegar e punho), devido quebra de ampolas de vidro para o preparo de medicamento.

Nenhum profissional acidentado fazia uso de luvas, sapato fechado, avental, óculos protetores e máscara. Apenas as luvas e as máscaras eram disponibilizadas pelos serviços para todos trabalhadores.

\section{DISCUSSÃO E CONCLUSÃO}

A epidemiologia de acidentes com MB é usualmente realizada a partir de questionários ou revisão de prontuários, o que difere do modo dos acidentes aqui relatados que aconteceram na presença de um pesquisador, em período, relativamente, curto de observação. Isto torna difícil a comparação com outras pesquisas.

A negligência no uso dos equipamentos de proteção implica em responsabilidades institucionais e individuais, os serviços devem disponibilizar esses dispositivos conforme os riscos envolvidos e aos trabalhadores cabe o uso adequado e conservação dos mesmos (11). Para todos os casos, as luvas deveriam ter sido adotadas, pois são indicadas para os procedimentos realizados (12) e minimizaria o risco de contaminação nos casos de 1 a 7 .

O sapato fechado não previsto dentre as medidas nas Precauções Padrão (4) e recomendado no Brasil como item de proteção obrigatório (11) minimizaria o risco nas exposições dos casos 6 e 7 .

Os óculos de proteção e a máscara são indicados apenas, quando respingos de sangue são esperados no referido procedimento (12). Diante disso, questiona-se a probabilidade de a decisão pelo uso de determinados equipamentos de proteção ficar sob a responsabilidade de cada profissional de saúde, especialmente, nas unidades de urgência e emergência onde a demanda exige processos 
decisórios imediatos que requerem desses profissionais a priorização do cuidado com o outro.

O caso 8 (exposição de pele e mucosa a vômito e secreção pulmonar) parece uma situação inusitada, entretanto, em um estudo onde $11,7 \%$ dos acidentes com MB envolveram a região dos olhos de profissionais da equipe de enfermagem, em três casos $(8,6 \%)$ havia secreção pulmonar envolvida (1). Nesse caso o risco foi potencializado pelo não uso de óculos protetores, equipamento não indicado para administração de injetáveis (12). Uma revisão sistemática da literatura apontou poucos relatos de acidentes-ocupacionais envolvendo $\mathrm{MB}$ em mucosas, durante acessos venosos periféricos e ressaltou a importância da notificação para realização de um diagnóstico confiável (13).

Os acidentes com material biológico relatados neste estudo reforçam o princípio das Precauções Padrão (PP) de incorporar medidas preventivas, como o uso de equipamentos de proteção, à prática laboral, com vistas à redução desse agravo em todos os momentos assistenciais, independente do diagnóstico presumível ou conhecido do paciente (4). Princípio contrário do relatado em um estudo onde conhecer o status sorológico do paciente foi uma condição, em que o profissional de saúde se baseou para modificar seu comportamento e aderir ou não a medidas preventivas, como aos equipamentos de proteção (14).

Enfatizamos a importância da aplicação das Precauções Padrão nos de urgência e emergência, móveis e fixos, pelas características peculiares envolvidas. Para esses setores a recomendação para profissionais não seria a de usar todos os equipamentos de proteção durante qualquer atendimento? Inclusive durante a administração de injetáveis, momento em que pode haver alterações no quadro do paciente e ocasionar, dentre outras reações, vômito; tontura; agitação; síncope aumentando a possibilidade de exposição.

Apesar de nenhum profissional inter- romper o atendimento imediatamente após o acidente, a maioria realizou corretamente os cuidados locais pós-exposição $(5,12)$. Exceto no caso 8 em que não houve a lavagem exaustiva da mucosa ocular, conforme recomendado $(5,12)$.

A não notificação do acidente pelo profissional, no caso que envolveu mucosa (caso 8), leva-nos a questionar sobre a percepção do risco que permeia essas exposições. Pode haver desinformação ou desinteresse dos profissionais da área da saúde em relação aos aspectos epidemiológicos envolvidos nessas situações. Porém, é fato que, a gravidade e as consequências dos acidentes, assim como as possibilidades de intervenção, somente poderão ser avaliadas com a notificação e o acompanhamento sorológico preconizados.

A associação entre o tipo de acidente e sua notificação é evidenciada pela literatura, estudo identificou que a taxa de notificação era maior quando o profissional considerava o acidente como de maior gravidade (15).

Como iniciativa que busca modificar o quadro atual de subnotificação dos acidentes envolvendo $\mathrm{MB}$ no país destaca-se a atualizada Portaria No 104 que inclui o acidente com exposição a MB relacionado ao trabalho, na Lista de Notificação Compulsória no Brasil, e obriga o registro, eletrônico, no Sistema de Informação de Agravos de Notificação (16). Além disso, normativas brasileiras estabeleceram a obrigatoriedade de substituição de materiais perfurocortantes por aqueles com dispositivos de segurança (17). Esses dispositivos não estavam presentes nas unidades, entretanto a coleta ocorreu no prazo de adaptação previsto na Portaria (18) para a substituição.

Chama atenção a relação risco físico e biológico observado no caso 8, que além da exposição às secreções em mucosa ocular houve a contato na região das mãos e braços e o profissional apresentava cortes visíveis nos dedos, provocados pela quebra de ampolas ao preparar medicamentos, não usava luvas e não lavou, exaustivamente, os locais afeta- 
dos, limitou-se a um breve enxágue.

Todas as vítimas foram individualmente orientadas nos momentos imediatos que sucederam os acidentes, de modo especial quanto às condutas pós-exposição e necessidade de notificação do ocorrido, e posteriormente, receberam um exemplar de um manual de condutas frente a acidente com MB (19).

A oportunidade de presenciar acidentes com MB por trabalhadores de enfermagem nas etapas finais do sistema de medicação em unidades de urgência e perceber suas particularidades contribuíram para a compreensão do quanto essas unidades carecem de uma cultura preventiva, pois um procedimento rotineiro pode representar risco para os trabalhadores. O planejamento e implementação de estratégias educativas devem ser direcionadas por um diagnóstico situacional propondo medidas aplicadas à prática que tenham significado para esses trabalhadores e conduzam a adoção de comportamentos seguros, destaca-se que os sistemas de feedback mostram-se eficazes (5).

A maioria dos acidentes foi por sangue nas mãos dos trabalhadores e três envolveram vômito sinalizando para uma relação da condição de saúde do usuário que busca os serviços de urgência e emergência, especificamente na administração de medicamentos. O não uso dos equipamentos de proteção em todos os acidentes mostra uma subvalorização dessas barreiras por parte dos trabalhadores e ainda dos gerentes, já que nem todos estavam disponíveis.

Os dados sugerem que a notificação dos acidentes com MB permanece como um desafio à segurança dos trabalhadores. Tornar a notificação desse agravo compulsória no país não tem sido suficiente, parece haver uma avaliação pelo próprio trabalhador que orienta a sua tomada de decisão quanto às condutas, haja vista que todos procederam à limpeza local, mesmo que não tenha sido conforme o recomendado. Aspectos que merecem ser investigados.
É possível afirmar que o processo de trabalho nos setores de urgência e emergência requer um olhar diferenciado, que considere as suas especificidades, para o planejamento e implantação de uma cultura de segurança frente ao risco biológico.

O caráter observacional do estudo apesar de possibilitar o fornecimento de dados extremamente fidedignos, não permitiu investigar questões subjetivas, de análise do risco e razões da negligência ao uso de equipamentos de proteção. Questões que merecem ser investigadas.

A presença de um pesquisador/observador, externo ao grupo de trabalho, foi uma limitação do estudo que pode ter influenciado no comportamento das vítimas. Entretanto, o mais coerente seria pensar em uma previsão de alteração buscando condutas corretas, ainda assim medidas preventivas pré e pós-acidentes foram negligenciadas. Um importante caminho para a enfermagem, no contexto da segurança do trabalhador, é intervir utilizando questões existentes no cotidiano laboral desses profissionais, como aqueles referidos no presente estudo, tanto nos cursos de formação quanto nos programas de educação permanente. Novas e diferentes ferramentas podem ser aliadas nessa nova forma de se refletir acerca de comportamentos e de percepção de risco, como simulações e casos clínicos baseados na prática.

\section{REFERÊNCIAS}

1. Gourni P, Polikandrioti M, Vasilopoulos G, Mpaltzi E, Gourni M. Occupational Exposure to blood and body fluids of nurses at Emergency department. Health Science Journal. 2012; 6(1): 60-8.

2. Marziale MH, Rocha FL, Robazzi ML, Cenzi CM, dos Santos HE, Trovó ME. Organizational influence on the occurrence of work accidents involving exposure to biological material. Rev Lat Am Enferma- 
gem. 2013; 21(Spec No): 199-206.

3. Harris SA, Nicolai LA. Occupational exposures in emergency medical service providers and knowledge of and compliance with universal precautions. Am J Infect Control. 2010; 38: 86-94.

4. Siegel JD, Rhinehart E, Jackson M, Chiarello L, Healthcare Infection Control Practices Advisory Committee. Guideline for isolation precautions: preventing transmission of infectious agents in healthcare settings. Transmission-based precautions. Atlanta (GA): Centers for Disease Control and Prevention (CDC); 2007.

5. Rapparini C, Reinhardt EL. Manual de implementação: programa de prevenção de acidentes com materiais perfurocortantes em serviços de saúde. São Paulo: Fundacentro, 161p, 2010.

6. Amaral PM, Tavares-Neto J. Exposição ocupacional a material biológico por pessoal de saúde: construção e proposição de instrumentos avaliativos. Rev bras saúde ocup. 2010; 35(121): 131-47.

7. Soares LG, Sarquis LMM, Kirchhof ALC, Felli VEA. Multicausalidade nos acidentes de trabalho da Enfermagem com material biológico. Rev bras enferm. 2013; 66(6): 854-9.

8. Danna MF, Matos MA. Aprendendo a observar. São Paulo: Edicon. 2006.

9. Malta M, Cardoso LO, Bastos FI, Magnanini MMF, Silva CMFP. STROBE initiative: guidelines on reporting observational studies. Rev Saude Publica 2010; 44(3): 559-65.

10. Von Elm E, Altman DG, Egger M, Pocock SJ, Gøtzsche PC, Vandenbroucke JP, et al. Strengthening the Reporting of Observational Studies in Epidemiology (STROBE) statement: guidelines for reporting observational studies. BMJ. 2007; 335 (7624): 806-8.
11. Ministério do Trabalho e Emprego. Portaria No 485/GM de 11 de novembro de 2005. Aprova a Norma Regulamentadora No 32. Segurança e Saúde no Trabalho em Estabelecimentos de Saúde. Brasília (Brasil): Ministério da Saúde; 2005.

12. World Health Organization. WHO Best practices for injections and related procedures for toolkit 2010. p. 69.

13. Hadaway L. Needlestick injuries, short peripheral catheters, and health care worker risks. Infus Nurs. 2012; 35(3): 164-78.

14. Garus-Pakowska A, Szatko F. Percutaneous exposures in medical personnel. Med Pr. 2011; 62(5): 473-80.

15. Jagger J, Perry J, Parker G, Phillips EK. Blood exposure risk during peripheral I.V. catheter insertion and removal. Nursing. 2011; 41(5): 25-6.

16. Ministério da Saúde. Portaria No 104, de 25 de janeiro de 2011. Define as terminologias adotadas em legislação nacional, conforme disposto no Regulamento Sanitário Internacional 2005 (RSI 2005). Brasília (Brasil): Ministério da Saúde; 2011.

17. Ministério da Saúde. Portaria No 939 de 18 de novembro de 2008. Cronograma da implantação de materiais perfurocortantes com dispositivos de segurança. Brasília (Brasil): Ministério da Saúde; 2008.

18. Ministério da Saúde. Portaria No 1.342, de 9 de junho de 2011. Prorroga o prazo estabelecido no art. $4^{\circ}$ da Portaria $N^{o}$ 3.995/GM/MS, de 16 de dezembro de 2010. Brasília (Brasil): Ministério da Saúde; 2011.

19. Ministério da Saúde. Secretaria Municipal de Saúde. Centro de Referência em Saúde do Trabalhador de Goiânia. Exposição ocupacional a material biológico. Goiânia (Brasil): Ministério da Saúde; 2007. 